[Agr. Biol. Chem., Vol. 29, No. 2, p. 111 116, 1965]

\title{
Glyceride Structure and Biosynthesis of Natural Fats
}

\section{Part IV Biosynthetic Process of Triglycerides in Maturing Seed of Chinese Tallow Tree}

By Osamu Hrayama and Shingo Ohama

College of Agriculture, Kyoto Prefectural University, Kyoto

Received August 20, 1964

\begin{abstract}
Changes in lipid classes and water-soluble components in maturing seed of Chinese tallow tree were examined. Rapid oil production occurred at three stages of seed maturity. Stillingia oil syntheses in seed kern proceeded more rapidly than stillingia tallow on the kern at early stage. Analytical data for components in immature seed suggest that the pathway of seed oil synthesis is the same as Kennedy's pathway. However, the appearance of monoglycerides indicates the existence of a side pathway. From specific positional distribution of fatty acids in mono-, di-, and triglyceride from immature seed, synthetic process of glyceride structure was discussed.
\end{abstract}

In previous paper, ${ }^{11}$ biosynthetic process of triglycerides in maturing soybean seed has been investigated by measuring changes in the contents and composition of lipid and water-soluble components. A similar investigation was attemped on maturing seed of Chinese tallow tree (Sapium sebiferum Roxb.) in the present work. Chinese tallow tree accumulates solid fat (stillingia tallow) on the surface of seed kern, and liquid oil (stillingia oil) in the kern. It has been known that stillingia tallow is a vegetable fat which has relatively simple composition of triglyceride, and stillingia oil is a drying oil containing 2, 4-decadienoic acid as particular component acid. ${ }^{2)}$ These characteristics of the seed fat aroused our interest in study on their biosynthetic process.

\section{MATERIALS AND METHODS}

\section{Seed Preparation}

The seeds of Chinese tallow tree were collected eleven times from 15 th days till 142nd days after

1) O. Hirayama and K. Hujii, This Journal, 29, 1 (1965).

2) E. W. Eckey and L. P. Miller, "Vegetable Fats and Oils," Reinhold Publishing Co., 1954, p. 598 600. flowering, and sample number was given as shown in Table 1.

Extraction and Fractionation of Lipids and Water-soluble Components

The seeds were extracted two times with hot acetone under shaking. The extracts were combined, and evaporated to dryness to obtain crude stillingia tallow. The fat was further refined by re-extraction with diethyl ether and evaporation. The residual seed kern was crushed and homogenized with a mixture of chloroform-methanol $(2: 1, \mathrm{v} / \mathrm{v})$ using homogenizer. The same extraction was repeated once more. The residue was then extracted with hot water. Both solutions of total organic and aqueous extracts were transferred into separating funnel. After a few hours, resulting two layers were separated. The chloroform layer gave crude stillingia oil by removing the solvent. The aqueous layer was concentrated and dried under vacuum, the dry matter was extracted with $80 \%$ ethanol, and after evaporating the solvent, the extracted matter was weighed as water-soluble components. Crude stillingia oil was fractionated into five lipid classes of free fatty acid, mono-, di-, and triglyceride, and phospholipid by solvent separation and silicic acid column as described previously.1) Stillingia tallow was also separated into four classes of free acid, mono-, di-, and triglyceride. 
Whole seed oil in early stage was also prepared by the same procedure as that of stillingia oil.

\section{Analytical Methods}

All procedures for analyses of compositions in each lipid class and water-soluble components, and other analytical metoods such as lipase hydrolysis and periodate oxidation procedure were the same as described in previous paper."

\section{RESULTS}

It was observed that Chinese tallow tree produced a hard seed kern at about 50 days after flowering, and the isolated accumulation of stillingia tallow and stillingia oil occurred after 52 days (sample No. 4). Therefore, data of sample No. 1, 2, and 3 as described below represent those of whole oil mixture before they developed to stillingia tallow and stillingia oil. However, it seems that these whole oil mixtures correspond rather to the stillingia oil, as the oil formation curves (in Fig. 1) and the changes of acid values (in Table I) suggest.

The weight changes of glyceride, phospholipid, and water-soluble component per fresh thousand seeds during the ripening periods are shown in Fig. 1. It indicates that rapid production of whole oil occurred at three stages of maturity; the first stage of 37 to 52 days after flowering, the second of 67 to 82 days, and the third of 112 to 127 days. Although the amounts of stillingia tallow were smaller than stillingia oil at early stage, it increased more rapidly after 67 days, and

Table I. Chemical Properties of Seed OIL

\begin{tabular}{|c|c|c|c|c|c|c|c|}
\hline \multirow[b]{2}{*}{$\begin{array}{c}\text { Sample } \\
\text { No. }\end{array}$} & \multirow{2}{*}{$\begin{array}{l}\text { Days } \\
\text { after } \\
\text { flower- } \\
\text { ing }\end{array}$} & \multicolumn{2}{|c|}{ Stillingia } & oil & \multicolumn{2}{|c|}{ Stillingia } & talloow \\
\hline & & A.V. & I.V. & S.V. & A. V. & I.V. & S.V. \\
\hline 1 & 15 & & & & & & \\
\hline 2 & 22 & & & & & & \\
\hline 3 & 371 & 132.0 & & & & & \\
\hline 4 & 52 & 37.6 & & & 67.6 & & \\
\hline 5 & 59 & 4.7 & 179.5 & 182.9 & 13.9 & 43.7 & 215.7 \\
\hline 6 & 67 & 4.1 & 173.5 & 179.1 & 4.8 & 41.4 & 202.6 \\
\hline 7 & 82 & 2.7 & 172.2 & 172.3 & 2.8 & 37.4 & 222.4 \\
\hline 8 & 97 & 2.7 & 168.9 & 185.0 & 3.3 & 41.6 & 218.4 \\
\hline 9 & 112 & 2.3 & 165.4 & 182.9 & 3.7 & 42.8 & 213.9 \\
\hline 10 & 127 & 2.1 & 159.0 & 186.0 & 3.4 & 34.3 & 216.6 \\
\hline 11 & 142 & 3.1 & 169.4 & 185.3 & 4.2 & 37.1 & 197.0 \\
\hline
\end{tabular}

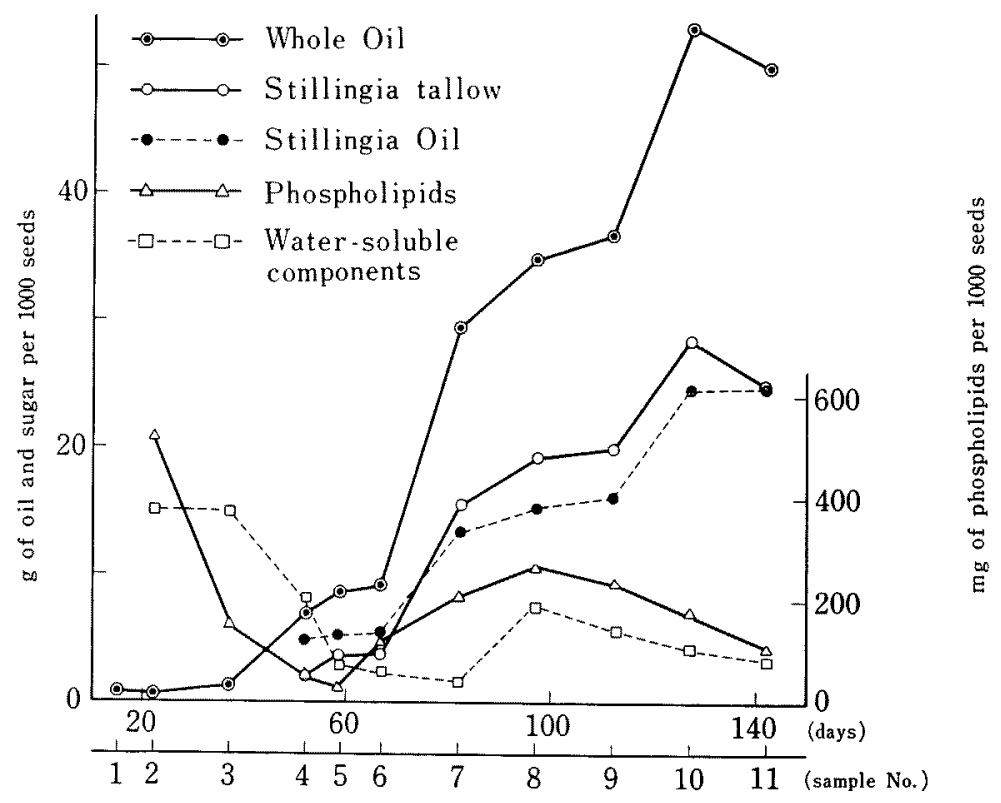

FIG. 1. Weight Changes of Lipids and Water-soluble Components in Maturing Seeds. 


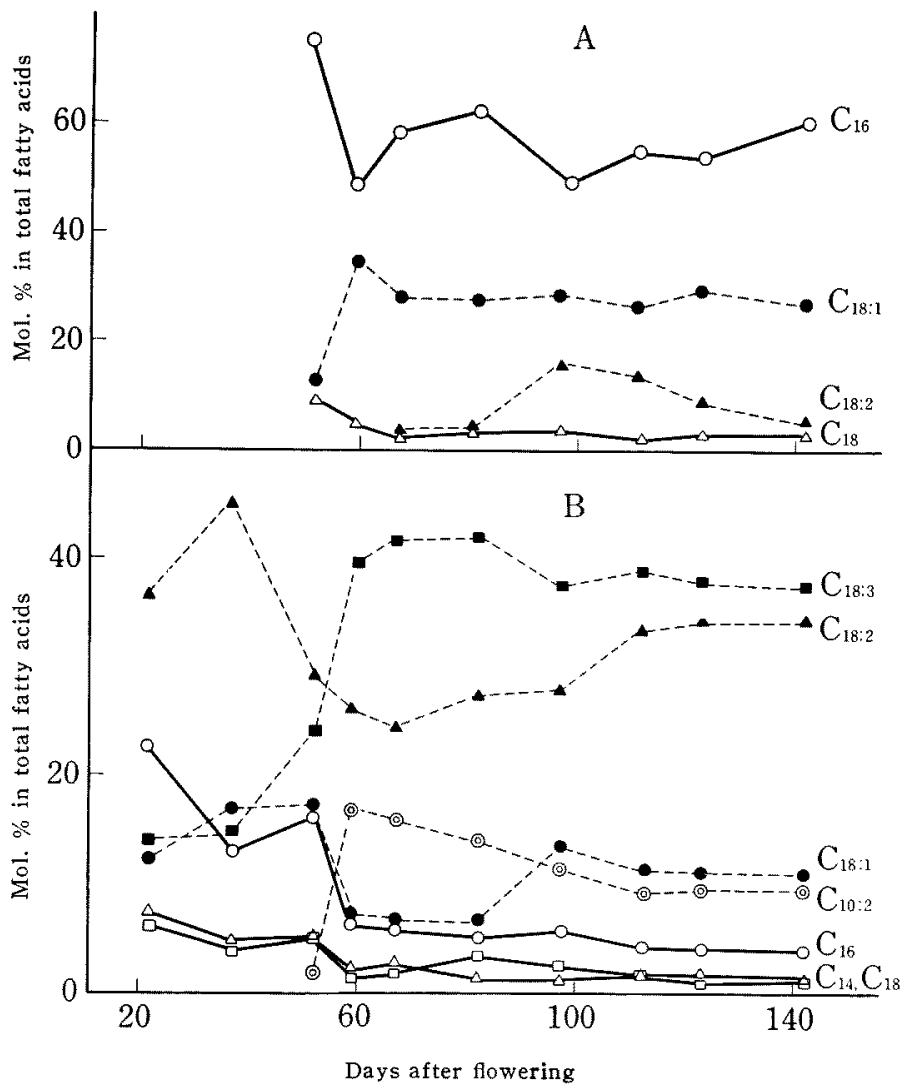

FIG. 2. Changes of Fatty Acid Composition in Stillingia Tallow (A) and Stillingia Oil (B) during the Ripening Periods.

Abbreviation: $C_{14}$, myristic, $C_{10}$, palmitic; $C_{18}$, stearic; $C_{10}: 2,2,4$-decadienoic; $C_{18}: 1$, oleic; $C_{18}: 2$ linoleic; $\mathrm{C}_{18}: 3$, linolenic.

at the last stage both the fat and oil reached to equal amounts. Phospholipid content changed with a characteristic curve, which might be connected with oil formation curve. A similar changes was also observed in the water-soluble component content.

Chemical properties of stillingia tallow and stillingia oil from each sample were presented in Table I. Iodine values and saponification values showed no significant change, but acid values suggested that large amounts of free fatty acids in immature seed disappeared at earlier stage in seed kern (stillingia oil) than on the surface of the kern (stillingia tallow).
Fig. 2 shows changes of fatty acid compositions of crude stillingia tallow and crude stillingia oil. In stillingia oil, relatively larger proportion of saturated acid contents at early stage decreased with maturation of seed. The changes of each unsaturated acid contents indicate a relation among co-occuring acids which partially suggest the conversion of oleic acid to higher unsaturated acids. Although 2, 4-decadienoic acid was absent in early immature seed, it was produced rapidly after 52 days and then followed nearly similar curve to that of linolenic acid. It was also observed in stillingia tallow that both acid 
contents of palmitic and oleic changed in an and monoglyceride contents showed maximinteresting relation each other during all um peak at 52 days after flowering and diripening periods.

The changes of free fatty acid and partial glyceride contents in early stages were shown in Fig. 3. It is noticed that free fatty acid glyceride content at 59 days; then they decreased and disappeared completely till 82 days. Monoglyceride and diglyceride in sample at 59 days were further analyzed by

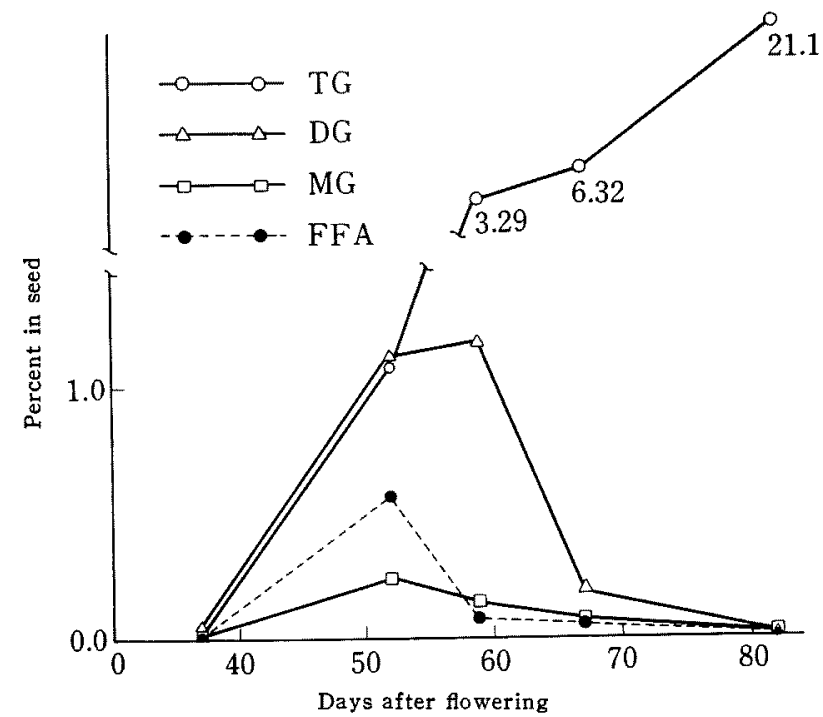

FIG. 3. Changes of Free Fatty Acid and Glyceride Contents in Immature Seed.

TG, triglyceride; DG, diglyceride; MG, monoglyceride; FFA, free fatty acid.

Table II. Positional Distribution of Fatty acids in Glyceride of Immature and Mature Seeds Obtained by Lipase Hydrolysis Procedure

\begin{tabular}{|c|c|c|c|c|c|c|c|c|c|c|c|c|}
\hline \multirow{3}{*}{$\begin{array}{l}\text { Fatty } \\
\text { acid }\end{array}$} & \multicolumn{3}{|c|}{ Sample 3} & \multicolumn{4}{|c|}{ Sample 6} & \multirow{3}{*}{$\mathrm{MG}$} & \multicolumn{3}{|c|}{ Sample II } & \\
\hline & \multicolumn{2}{|c|}{ TG } & \multirow[t]{2}{*}{ MG } & \multicolumn{2}{|c|}{ TG } & \multicolumn{2}{|c|}{ DG } & & \multicolumn{2}{|c|}{ TG 1} & TG 2 & \\
\hline & $\begin{array}{l}\text { Outer } \\
\text { chain }\end{array}$ & $\begin{array}{l}\text { Inner } \\
\text { chain }\end{array}$ & & $\begin{array}{l}\text { Outer } \\
\text { chain }\end{array}$ & $\begin{array}{l}\text { Inner } \\
\text { chain }\end{array}$ & $\begin{array}{l}\text { Outer } \\
\text { chain }\end{array}$ & $\begin{array}{l}\text { Inner } \\
\text { chain }\end{array}$ & & $\begin{array}{l}\text { Outer } \\
\text { chain }\end{array}$ & $\begin{array}{l}\text { Inner } \\
\text { chain }\end{array}$ & $\begin{array}{l}\text { Outer } \\
\text { chain }\end{array}$ & $\begin{array}{l}\text { Inner } \\
\text { chain }\end{array}$ \\
\hline $14: 0$ & 6 & 1 & 4 & 11 & & 15 & 5 & 30 & 1 & & 2 & 1 \\
\hline $16: 0$ & 21 & 2 & 31 & 59 & 25 & 71 & 14 & 70 & 13 & 2 & 91 & 3 \\
\hline $18: 0$ & 8 & 1 & 10 & & & & & & 7 & & 4 & 2 \\
\hline $18: 1$ & 12 & 15 & 13 & 25 & 73 & 14 & 67 & & 11 & 15 & 3 & 84 \\
\hline $18: 2$ & 38 & 62 & 29 & 5 & 2 & & 14 & & 27 & 45 & & 10 \\
\hline $18: 3$ & 16 & 19 & 15 & & & & & & 35 & 37 & & \\
\hline $10: 2$ & & & & & & & & & 5 & 1 & & \\
\hline$S$ & 35 & 4 & 45 & 70 & 25 & 86 & 19 & 100 & 21 & 2 & 97 & 6 \\
\hline $\mathrm{U}$ & 65 & 96 & 55 & 30 & 75 & 14 & 81 & & 79 & 98 & 3 & 94 \\
\hline
\end{tabular}

Sample 3 (whole oil), sample 6 (stillingia tallow), and sample 11 (TG 1, stillingia oil; TG 2, stillingia tallow) were separated into monoglyceride (MG), diglyceride (DG), and triglyceride (TG) on silicic acid column. Di- and triglyceride fraction obtained were hydrolyzed by pancreatic lipse. The composition of the resulting free fatty acids (outer chains) and monoglyceride-fatty acids (inner chains) were determined. $S$, total saturated; $U$, total unsaturated; $10: 2,2,4$-decadienoic acids. 


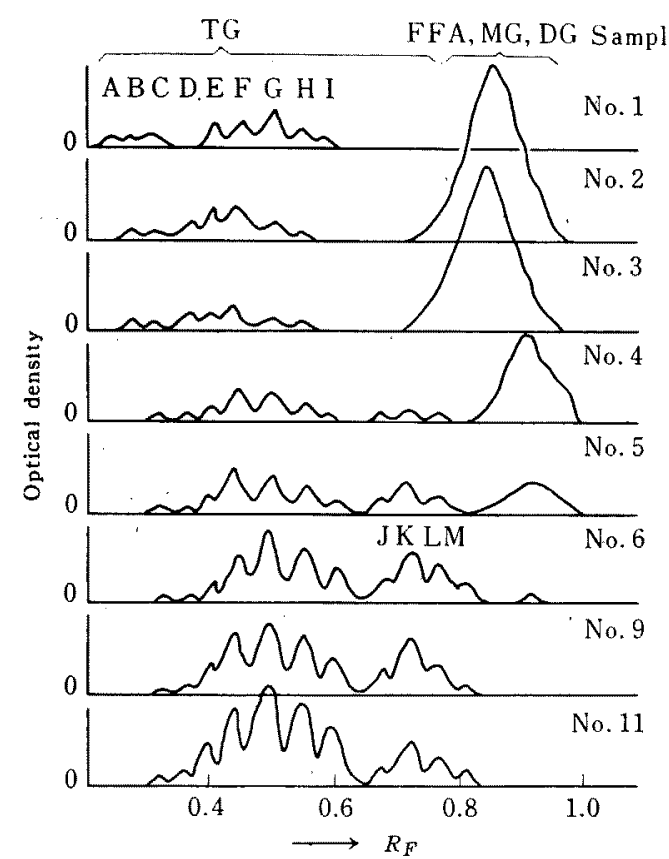

FIG. 4. Densitometer Curves of Paper Chromatograms of Stillingia Oil Glycerides.

Stillingia oils (sample No. 1 11) were run on the paper impregnated with silicon oil using acetone-acetic acid $(1: 4)$ as the developing solvent. The chromatograms were visi ualized with aqueous solution of $\mathrm{KMnO}_{4}$, and measured by densitometer. Main triglycerides in spots are as follows: A SSO, B Soo, C SOLe, D OOle, E OLele, F OLeLn, G LeLeLn, H LeLeLn, I LnLnLn, J DdOLe, K DdLeLe, L DdLeLn, M DdLnLn, S, saturated, O, oleic, Le, linoleic; Ln, tinolenic; Dd, 2,4-decadienoic, FFA, free fatty acid; $M G$ monoglyceride; $D G$, diglyceride; $T G$, triglyceride.

periodate oxidation procedure and thin-layer chromatography to determine the proportions of their isomers. The results suggest that the monoglycerides were composed of $72 \%$ of 1 isomer and $28 \%$ of 2 -isomer, and the diglycerides were mainly 1,2 -isomer.

Changes of triglyceride compositions in seed kern during the ripening periods are presented in Fig. 4, which show densitometer curves of paper chromatograms of triglycerides. The triglycerides in the earliest immature seed were divided into two groups of highly unsaturated and lowly unsaturated glycerides. The former group decreased and the latter increased with maturation transferring to higher PG value glyceride. New triglycerides containing 2, 4-decadienoic acid appeared after 52 days (sample No. 4), and somewhat later triglyceride composition in immature seed reached to nearly complete one (sample No. 6). Thereafter the amounts of stillingia oil increased to about four times without great change of glyceride composition. Fig. 4 also shows that a large proportion of partial glycerides and free fatty acids in stillingia oil at early stage decreased and disappeared completely at later stage.

Positional distributions of fatty acids in glyceride molecule of sample No. 6 and No. 11 were determined by the procedure of lipase hydrolysis. The results were given in Table II. It shows that the triglycerides in early immature seed have larger proportion of saturated acids at 1, 3-positions of glycerol molecule than at 2-position as well as the triglycerides in fully mature seed. The pattern of the fatty acid distribution in diglycerides in immature seed also corresponded to that of the triglyceride. Component acids of the monoglycerides in immature seed was composed of only saturated acids, which was

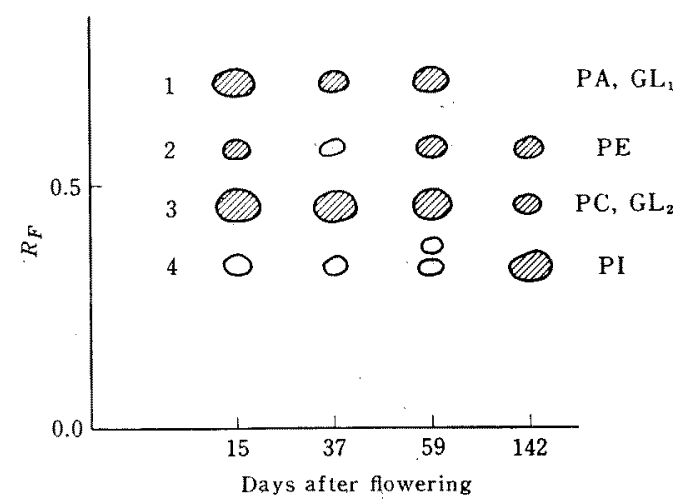

FIG. 5. Paper Chromatograms of Phospholipids in Immature Seed.

Samples were run on silicic acid-impregnated paper using disobutylketone-acetic acid-water $(45: 20: 3)$ as the solvent. The chromatograms were visiualized in aqueous solution of $\mathrm{KMnO}$.

PA, phosphatidic acid; PC, lecithin; PI, phosphatidyl ino. sitol; GL, glycolipid.

Blank spots show smaller amount of lipids. 
more similar to outer chains than inner chains of di- and triglycerides as described above.

Phospholipids from each sample were separated by silicic acid column and paper chromatography. Fig. 5 shows chromatograms by silicic acid-impregnated paper. Each spot was identified by stain behaviour, $R_{F}$ values of authentic materials, and deacylation products derived from each fraction which was obtained by silicic acid column. The results indicated that phospholipid fraction from immature seed was composed of phosphatidic acid (spot 1), phosphatidyl ethanolamine (spot 2), lecithin (spot 3), and phosphatidyl inositol (spot 5), with a large portion of glycolipids (spot 1 and 3). Fig. 5 illustrated that more lipids from spots 1 and 3 than phosphatidyl inositol were found in immature seed, whereas the reverse situation was observed in mature seed.

Paper chromatographic analyses of watersoluble components revealed that $\alpha$-glycerophosphate, sucrose, and other unknown phosphate and sugar are present in immature seed.

\section{DISCUSSION}

Weight changes (Fig. 1) of seed oil of Chinese tallow tree showed that the seed oil was not produced with continuous increase during maturation periods but it was produced rapidly at the isolated three stages. The stepwise oil formation, which was also observed in characteristic changes of fatty acid composition (Fig. 2), was similar to that of soybean seed as described previously. It appears (From Fig. 1 and Table I) that the oil in seed kern (stillingia oil) is produced more rapidly than the fat on the kern (stillingia tallow) at early stage and the accumulation process of the former oil proceeds slightly faster as compared with that of the latter fat.

3) E. J. Barron and P. K. Stumpf, Biochim. Biophys. Acta, 60, 329 (1962).
Although changes in fatty acid compositions suggest possible conversion of oleic acid to more highly unsaturated acids, no direct evidence was obtained. It is noticed that 2, 4-decadienoic acid appeared at later stage than other all fatty acids, and then increased rapidly during the period in which linolenic acid was produced remarkably.

Characteristic changes of contents and compositions of diglyceride, phospholipids, and water-soluble components in immature seed may be taken to support the following pathway to triglyceride: sugar $\longrightarrow \alpha$-glycerophosphate $\longrightarrow$ phosphatidic acid $\longrightarrow$ diglyceride $\longrightarrow$ triglyceride. The discovery that diglyceride was mainly composed of 1,2-isomer also give an additional suggestion for the occurrence of Kennedy's pathway. Original monoglycerides in immature seed which were considered to be almost 1-isomer, changed in content like the intermediate such as diglyceride, and their fatty acid composition was similar to those of outer chains of di- and triglyceride in the same sample. To our interest, the change of monoglyceride content is similar to that reported by Barron and Stumpf, ${ }^{3)}$ who studied on the biosynthesis of triglycerides by avocado-mesocarp enzymes. In the study they presume that the acylation of glycerolphosphate is a two-step process, and the lysophosphatidic acid produced is transformed to monoglyceride. If this assumption is true in the present seed oil formation, the data of positional distribution of fatty acids in the partial glycerides will indicate that the first acylation of glycerolphosphate occurs at $\alpha$ position with more portion of saturated acids and then the second acylation at $\beta$-position with more portion of unsaturated acids. These explanation may be also applied for data of soybean seed as described previously. 